\title{
Transport Properties of Concrete
}

\section{Peter Claisse}

Coventry University, Coventry CV1 5FB UK

"Peter Claisse graduated with a degree in Physics from Oxford University and then spent the next 9 years working as a Civil Engineer on major UK construction sites including 4 years on the Torness nuclear power station. After obtaining a $\mathrm{PhD}$ in Civil Engineering he then went to the AEA Technology Harwell laboratory for 3 years to work on Nuclear waste containment. He has been at Coventry University for the last 12 years teaching Civil Engineering Materials and researching transport processes in construction materials, waste containment, and the use of waste materials in construction."

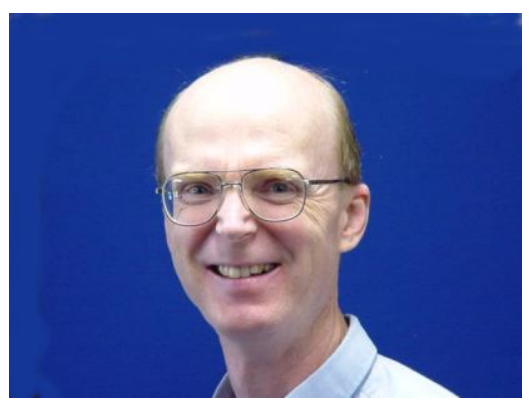

\begin{abstract}
In this paper the main transport processes that take place in concrete are described. For each process a brief introduction to the physical mechanism is developed by giving examples of how they can contribute to the deterioration of concrete structures by, for example, enabling salt to reach the reinforcement. An example of the practical use of the theory is taken from the design of a waste containment barrier.
\end{abstract}

\section{Introduction}

There are many different processes that may cause deterioration of a concrete structure including, for example, reinforcement corrosion, sulphate attack and frost damage. The common characteristic with all of these processes is that they require something to be transported into the concrete from outside or out from inside the concrete. Even alkali-silica reaction, which is a reaction between components which are already present inside a structure, requires water for the reaction to take place. In order to understand how to improve the 
durability of structures it is therefore essential to have a clear understanding of the different transport processes. These may take place in sound or cracked concretes but, even where cracks are present, nothing will move without a transport process.

In this paper the four main transport processes are described: Pressure driven flow, diffusion, electromigration and thermal migration. In addition to the external factors (such as an applied pressure) which may cause the transport there are also three significant processes which cause transport and are internal to the concrete: Adsorption may stop it and capillary suction and osmosis may increase it. These are also described.

The aim of the paper is to show how a systematic scientific evaluation of the transport properties of concrete can contribute to the design and construction process and reduce the whole-life cost of structures.

Before considering the processes in detail the exact nature of what is being transported must be defined. Damage may be caused to concrete by water itself, or chemicals dissolved in water. Many molecules will dissociate into two separate parts (ions) when they are in solution with each part carrying an opposite charge. For example common salt (sodium chloride, $\mathrm{NaCl}$ ) will dissociate into $\mathrm{Na}^{+}$and $\mathrm{Cl}^{-}$and hydrated lime (calcium hydroxide, $\mathrm{Ca}(\mathrm{OH})_{2}$ ) will dissociate into $\mathrm{Ca}^{++}$and $\mathrm{OH}^{-}$. These ions may move in two different ways. The water itself will move with the ions in it or the ions may move through the water. Thus the transport processes may cause damage both by movement of water or by ionic movement in the water.

It is necessary to make a clear distinction between absorption and adsorption. The term absorption is used to describe processes such as capillary suction and osmosis which may draw water into concrete. Adsorption is the term used for all processes which may bind an ion (temporarily or permanently) in the concrete and prevent it from moving. These processes may be chemical reactions or a range of physical surface effects.

There have been numerous significant publications on the transport processes. They were generally first documented by the end of the $19^{\text {th }}$ century and applied to concrete by the middle of the $20^{\text {th }}$. For further discussion readers are referred to Neville (1995) or Illston (2001) 


\section{The transport processes}

Permeability is defined as the property of concrete which measures how fast a fluid will flow through concrete when pressure is applied. In some types of structure, such as dams and tunnel lining there may be an external water pressure but in other it may be absorption processes which create pressure differentials.

Geotechnologists will define permeability in terms of the head of water on each side of the sample in meters and would find typical value for the coefficient of permeability of concrete is $10^{-12} \mathrm{~m} / \mathrm{s}$ (the name "hydraulic conductivity" is also used for this). Scientists will include the viscosity of the fluid in the calculation and define permeability in terms of the pressures on each side and would find typical value for the intrinsic permeability of concrete is $10^{-19} \mathrm{~m}^{2}$. The difference between these may be seen to be 7 orders of magnitude so care must be taken when interpreting results to ensure the correct units are used.

The significance of a high permeability to a structure may not simply be the ability of water to get into it; the more significant damage may come from chlorides and sulphates dissolved in the water which can then damage the reinforcement and the concrete itself.

Diffusion is a process by which an ion can pass through saturated concrete without any flow of water. Diffusion is driven by concentration gradient. If a strong solution is in contact with a weak solution they will both tend towards the same concentration. Thus, for example, if a pile of salt is placed in one corner of a container full of water diffusion will be the process that ensures that when the salt has dissolved it will assume a uniform concentration throughout the water.

In figure 2 we intuitively know that as the salt dissolves into the water it will assume an equal concentration at all points throughout the liquid on one side and it is easy to see that there is a mechanism that will take it through the concrete and eventually give the same concentration throughout. By the same mechanism ions which are present in the pore water of the concrete will diffuse out and also assume an equal concentration throughout the liquid.

Moisture diffusion will take place in a gas when the concentration of water vapour is higher in one region than another. This mechanism will enable water to travel through the pores of unsaturated concrete. 
It should be noted that the ions will generally diffuse in pairs with equal and opposite charges. If they do not do this they will build up an electrical potential which will cause them to electromigrate back together (see below).

Electromigration occurs when and an electric field (voltage difference) is present. This may be derived from an external source such as leakage from a direct current power supply but is also frequently caused by the electrical potential of pitting corrosion on reinforcing steel. If an electric field is applied across the concrete in figure 3 the negative ions will move towards the positive electrode.

Electromigration can be measured from the electrical resistance of the concrete because it is the only mechanism by which concrete can conduct electricity. Because it is conducted in this way, rather than with electrons as in a metal, direct current will carry chloride ions into the concrete or possibly hydroxyl ions out of it. This mechanism is used in the desalination process in which a positive voltage is applied to the surface of a concrete structure in order to extract the chlorides from it. This process has the added advantage of applying a negative voltage to the reinforcing steel which will directly inhibit corrosion in the same way as cathodic protection. Unfortunately it also has the disadvantage of removing other negative ions from the concrete which may cause problems in practice.

Thermal Gradient: Water will move from hot regions to cold regions in solids. The rate at which it moves will depend on the permeability of the solid. This process is independent of and additional to the drying process (evaporation) which will take place on exposed surfaces which are hot. Similarly in saturated concrete ions in hotter water will migrate towards colder regions. The mechanism is shown in figure 4 and depends on probability. An ion or molecule which is moving faster on the hot side has a greater probability of crossing the sample than one on the cold side.

The most obvious situation when this process may occur is when a concrete structure which has been contaminated with de-icing salt heats up in sunlight. The salt saturated water in the surface pores will migrate rapidly into the structure. Even if this does not reach the steel by this mechanism the salt may diffuse the remaining distance. 


\section{Processes which increase or reduce the transport.}

Adsorption: When considering transport of ions in a porous material it is essential to consider adsorption at the same time because in many situations the bulk of the ions which enter into a barrier will be adsorbed before they reach the other side. Adsorbed ions are fixed into the matrix in various ways and are unable to move and therefore unable to cause any deterioration.

When the chloride concentration in a concrete sample is measured there are various different systems that can be used:

- If the "acid soluble" concentration is measured by dissolving the sample in acid this will extract all of the chlorides including those adsorbed onto the matrix.

Formatted: Bulleted + Level: $1+$ Aligned at: $0.63 \mathrm{~cm}+$ Tab after: 1.27 $\mathrm{cm}$ + Indent at: $1.27 \mathrm{~cm}$

- If the "water soluble" concentration is measured by leaching a sample in water only the ions in solution will come out (assuming the test is too short for adsorbed ions to dissolve). Alternatively "pore squeezing" or "pore fluid expression" can be used to squeeze the sample like an orange (using very high pressures).(Glasser 1992)

It can be argued that the ions in solution are the only ones that will cause corrosion and therefore the water soluble test is the most useful. Unfortunately, due to the possible release of bound chloride during the test and the effect of temperature and carbonation on the capacity factor, the acid soluble test is likely to give more repeatable results. The ratio of the solid to liquid concentrations is known as the capacity factor. In concrete the adsorption is normally on the cement (binding on the aluminate phases). Thus the capacity factor will be proportional to the cement content. It will probably be higher if Pulverized Fuel Ash is used.

A simple approximation of the amount of material which is adsorbed onto the matrix may be obtained by assuming that at all concentrations it is proportional to the concentration of ions in the pore fluid (note that this implies that the adsorption is reversible). Thus the capacity factor is a constant for all concentrations. This approximation is useful for modelling but it works best for low solubility ions, unlike chlorides which have a solubility of about $10 \%$. For chlorides there will still be a link between the number in solution and the number adsorbed but it will not be a very linear relationship. (Glasser 1992)

It may be seen that a high capacity factor will make the concentration change much more slowly - i.e. if chlorides are penetrating into a wall it will delay the start of corrosion of the steel. 
Diffusion with adsorption: Because there are two different ways of measuring concentration in an adsorbing system there are also two different ways of measuring diffusion:

- The apparent diffusion coefficient (which is what can be measured by testing the solid using measurements of total concentration) is defined from measurements of total concentration. (Atkinson and Nickerson 1984). This is also known as the "effective" diffusion coefficient.

- The intrinsic diffusion coefficient (which is the diffusion coefficient for the pore solution) is defined from measurements of the water soluble concentration. (Atkinson and Nickerson 1984)

Capillary Suction: Capillary suction occurs in fine voids (capillaries) with wetting surfaces and is caused by surface tension. In the experiment shown in figure 5 water rises higher up a smaller diameter glass capillary tube and this shows how this mechanism has greatest effect in systems with fine pores. This leads to the situation that concretes with finer pore structures (normally higher grade concretes) will experience greater capillary suction pressures. Fortunately the effect is reduced by the restriction of flow by generally lower permeabilities.

A good demonstration of the power of capillary suction in concrete can be observed by placing a cube in a tray of salt water and simply leaving it in a dry room for several months. The water with the salt in it will be drawn up the cube by "wicking" until it is close to an exposed surface and can evaporate. As this happens the near-surface pores fill up with crystalline salt which will eventually achieve sufficient pressure to cause spalling. This mechanism of damage by salt crystallisation is common in climates where there is little rain (not England!).(Illston 2001 p210)

Osmosis: Osmosis depends on what is called a semi-permeable membrane. This is a barrier through which the water can pass but material dissolved in it cannot pass as easily. An example is the surface layer of concrete which will permit water to enter but restrict the movement of the lime dissolved in the pore water. The osmotic effect causes a flow of water from the weak solution to the strong solution. Thus water on the outside of concrete (almost pure, i.e. a weak solution) is drawn into the pores where there is a stronger solution. The process is illustrated in figure 6. If two solutions were placed either side of a barrier as shown the level in one of them 
would rise, although in practice this would be very difficult to observe because concrete is permeable and the liquid would start to flow back as soon as a pressure difference developed.

If different solutions are present on each side of the sample osmosis will still take place even if the concentrations are the same. The direction of flow will depend on their relative "osmotic coefficients". Osmosis could be a significant process for drawing chlorides and sulphates into concrete. Having entered the concrete they may move further in by diffusion.

\section{Controlling parameters}

Having defined the transport processes the key questions are how they may be controlled and how they will affect the durability of a structure. In figure 7 the left hand column shows a number of factors which we may expect to affect the properties of concrete. They do not, however, directly affect the transport properties and the next column shows the actual internal properties which they are likely to affect. These include microcracks, the chemistry and also the "formation factor" for the pores which is a measure of how many direct paths there are through the pore structure. The third column shows the transport properties and the final column shows the deterioration processes that we want to inhibit. Thus we change something in the left hand column in the hope that it will affect the next two columns and finally get a result in the last column. The complexity of this situation explains why it is so difficult to achieve durability in a structure. For structural analysis the relationship between what we do and the results that we get is defined by quite precise equations. For durability even moving from one column to the next in the table is unfortunately only possible by using experimental data which is often difficult to interpret. There are, however, numerous significant relationships on the table which may be exploited.

The green arrows start from the water to cementitious ratio and the curing conditions. If the water content is kept low this will reduce the number of capillary pores that contain it and the overall porosity will be reduced. Similarly good water-retaining curing will promote full hydration and the resulting products will fill many of the pores and reduce the porosity. Following across the table shows that this will reduce the pressure driven flow and the diffusion. The pressure driven flow then causes frost attack (in combination with capillary suction) and diffusion is a key mechanism in reinforcement corrosion by carbonation or chloride ingress. 
The black arrows show that the cement type will be the major factor determining pore fluid chemistry. In particular the use of a pozzolanic material such as pulverized fuel ash or silica fume will reduce the amount of hydroxyl (lime) and alkali ions in solution. These are the main charge carriers and therefore the electromigration will be greatly reduced. The key effect of this is to reduce corrosion of reinforcement because this depends on the electromigration of these negatively charged ions from the cathode to the anode so they can combine with positive metal ions.

The red arrows show that the cement type will also be the key factor in determining the chemistry of the cement matrix that forms the structure of the hydrated paste. In particular if a sulphate resisting cement is used there will be few aluminates in it. This will, in turn, severely limit the ability of the matrix to adsorb chloride ions and they will thus remain free to cause corrosion.

There are very many other links in the table, indeed almost every factor in each column affects every factor in the next one to some extent and exploring each of them reveals methods that offer the potential to be exploited to improve durability.

It may be seen from the discussion above that reducing transport processes will normally improve durability. An exception to this is the deterioration of saturated concrete in fire. The Channel Tunnel linking England with France experienced a severe fire and photographs of the damage remarkably reveal apparently undamaged reinforcing steel with virtually no remaining concrete. In this incident the exceptionally low permeability of the concrete prevented the escape of steam from the pores of the saturated lining segments and caused them to literally explode. This phenomenon may be demonstrated by placing very low permeability concrete in a microwave oven. The only solution to this that is known to the author is to mix the concrete with polypropylene fibres that melt at high temperatures (Illston 2001 p210).

When considering figure 7 it must also be observed that building structures with concrete with low transport properties is of no use at all if the depth of the cover layer is not maintained. If the reinforcement is just a few millimetres below the surface nothing will protect it from the external environment.

\section{Example: Calculations for a waste containment barrier.}


The author is currently engaged in the design of a concrete barrier for waste leachate containment in landfills and the calculations for this illustrate a practical use of a knowledge of the processes. This barrier system is intended to replace current sand-bentonite barriers and is economically justified by the use of industrial wastes to make the concrete (Claisse et. al. 2003). The leachate above the barrier contains contaminants and the aim of the design is to minimise the output of them from the base.

Figures 8-11 show the influence of the physical and chemical properties of the concrete on the transport through the barrier. They were produced with a numerical model which applies equations for pressure driven flow, diffusion and adsorption (Claisse et al 2003) [ref].

Figures 8 shows the base case which is a $1 \mathrm{~m}$ head of leachate on a $1 \mathrm{~m}$ thick concrete barrier with a permeability of $10^{-9} \mathrm{~m} / \mathrm{s}$, a diffusion coefficient of $5 \times 10^{-10} \mathrm{~m}^{2} / \mathrm{s}$ and a capacity factor of 10 (typical values for concrete). The graph shows the concentration of contaminants across the barrier at different times. It may be seen that in the steady (final) state the concentration is at its maximum level throughout the barrier. This occurs because the flow is driven by pressure gradient and water with a high concentration of contaminants will flow all of the way through the barrier. Figure 9 shows the output of ions from the base of the barrier against time. The breakthrough time (158 years) is obtained by extrapolating the linear part of the curve back to the axis. The second line on the graph shows the situation with the permeability reduced to $10^{-}$ $12 \mathrm{~m} / \mathrm{s}$ (typical value for a very good concrete). The breakthrough time has only increased to 1049 years for the three order of magnitude decrease in the permeability. The reason for this can be seen in Figure 10 where the steady state concentration can be seen to show a linear decrease across the sample because diffusion is driven by the concentration gradient and can therefore never cause the concentrations to rise above these values.

Figure 11 shows the effect of changing the capacity factor for each of the examples shown above. It may be seen that this increases the breakthrough time far more effectively than reducing the permeability. The model has been validated by constructing trial cells, filling them with leachate and extracting samples of pore solution at various depths over a period of three years. With this validation it is now possible to use the knowledge of the transport properties to predict the performance of the barrier throughout its life. This makes it possible select materials 
for the concrete which will achieve the containment performance required by the regulatory authorities.

\section{Conclusion}

This paper defines the various transport mechanisms that allow deleterious ions and water to penetrate into concrete. Some guidelines are given to relate a concrete composition or mixture design with the transport and deterioration processes. It was shown that more than one factor in the concrete preparation can affect one deterioration process. Nevertheless, a good understanding of the various relationships allow an optimisation of a concrete to increase durability. An example using the concepts describe was given showing how the selection of the concrete permeability can increase in service life from 158 years to 1049 years but this could be achieved more easily by increasing the adsorption.

\section{References}

Atkinson A and Nickerson A K, "The Diffusion of Ions through Water-Saturated Cement” J.Mater Sci 19, 1984, 3068-3078

Claisse, P A Atkinson A, Ganjian E and Tyrer M., "Recycled Materials in Concrete Barriers" ACI publication SP212-59. Proc. $6^{\text {th }}$ Canmet/ACI conference on the Durability of Concrete, Thessaloniki, Greece, June 2003 pp.951-971.

Glasser F "Coexisting Solids and Aqueous Phase in Portland Cement". in Proc. RILEM worksop "Hydration and Setting of Cements" ed. Nonat A and Mutin C, Spon, 1992.

Illston J M, “Construction Materials”, E \& FN Spon, Chapman \& Hall, New York, $3^{\text {rd }}$ ed. 2001

Neville A M, "Properties of Concrete", Longman, Harlow, UK, $4^{\text {th }}$ ed. 1995. 


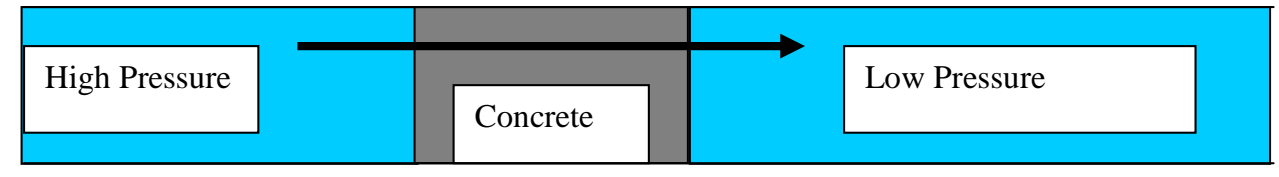

Fig. 1 Schematic diagram of pressure driven flow.

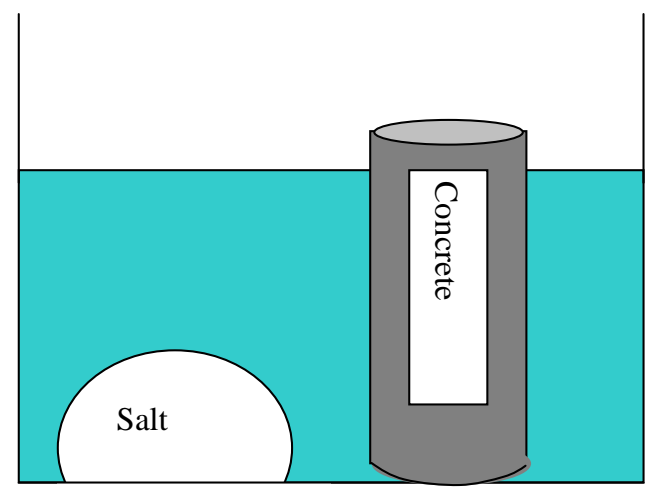

Fig. 2 Schematic diagram of diffusion. 


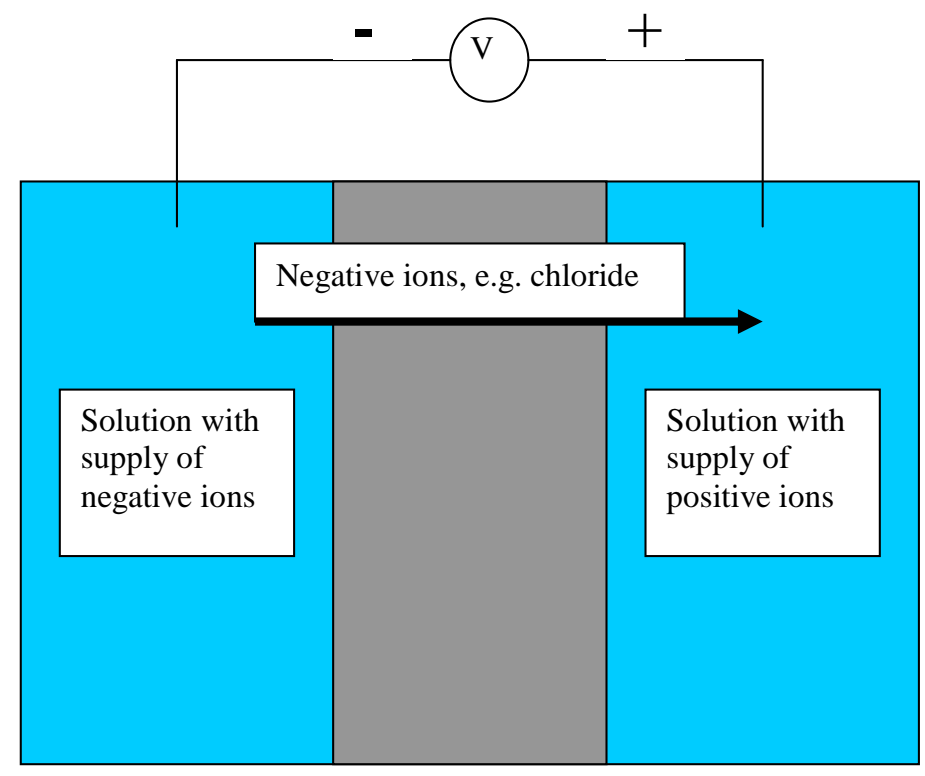

Fig 3 Schematic diagram of Electromigration

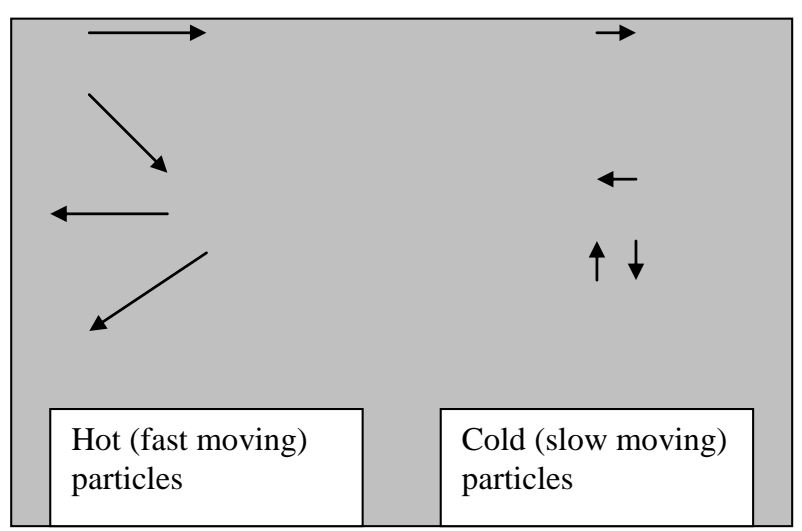

Figure 4 Schematic diagram of thermal migration. 


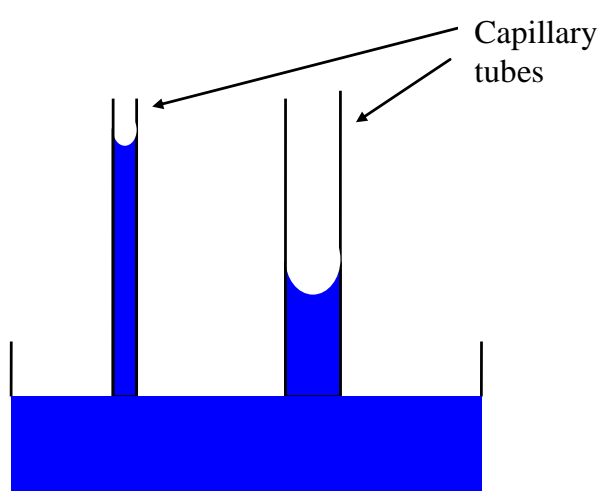

Figure 5 Capillary suction

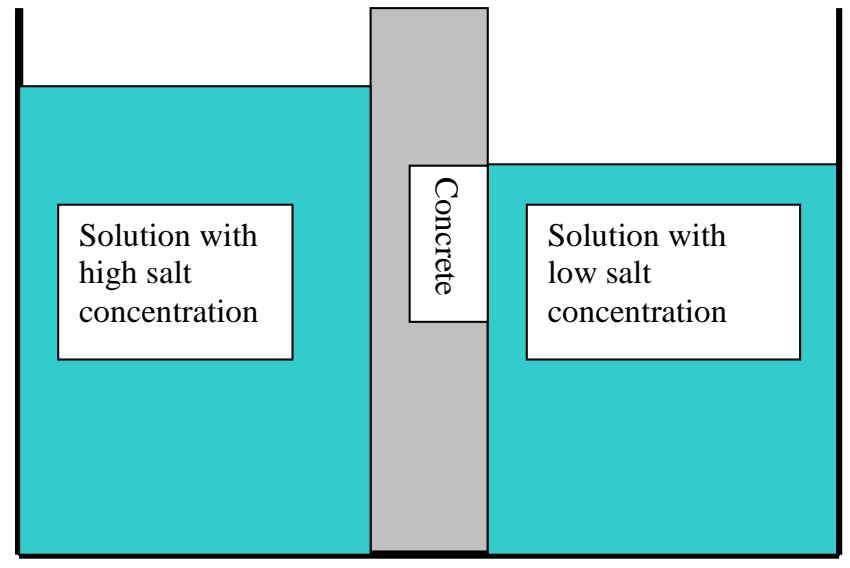

Figure 6 Osmosis 


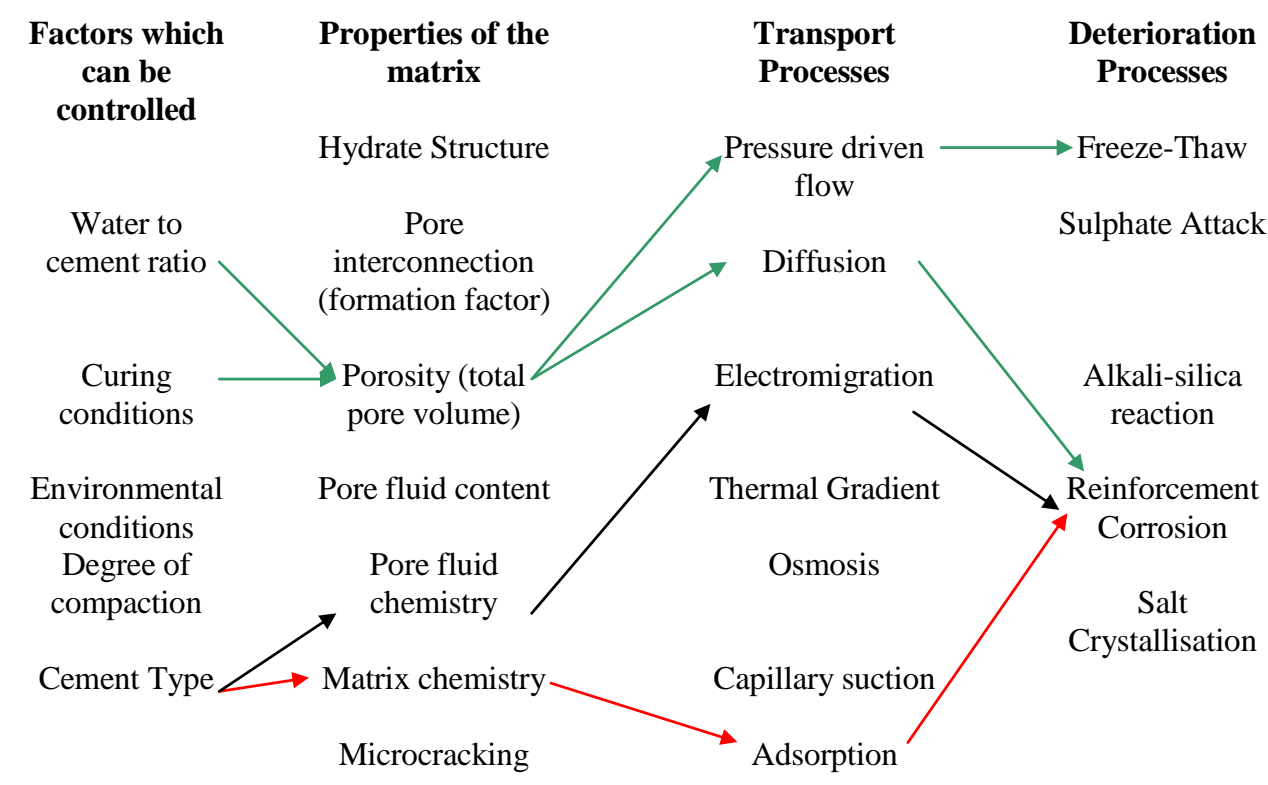

Figure 7 Factors affecting durability

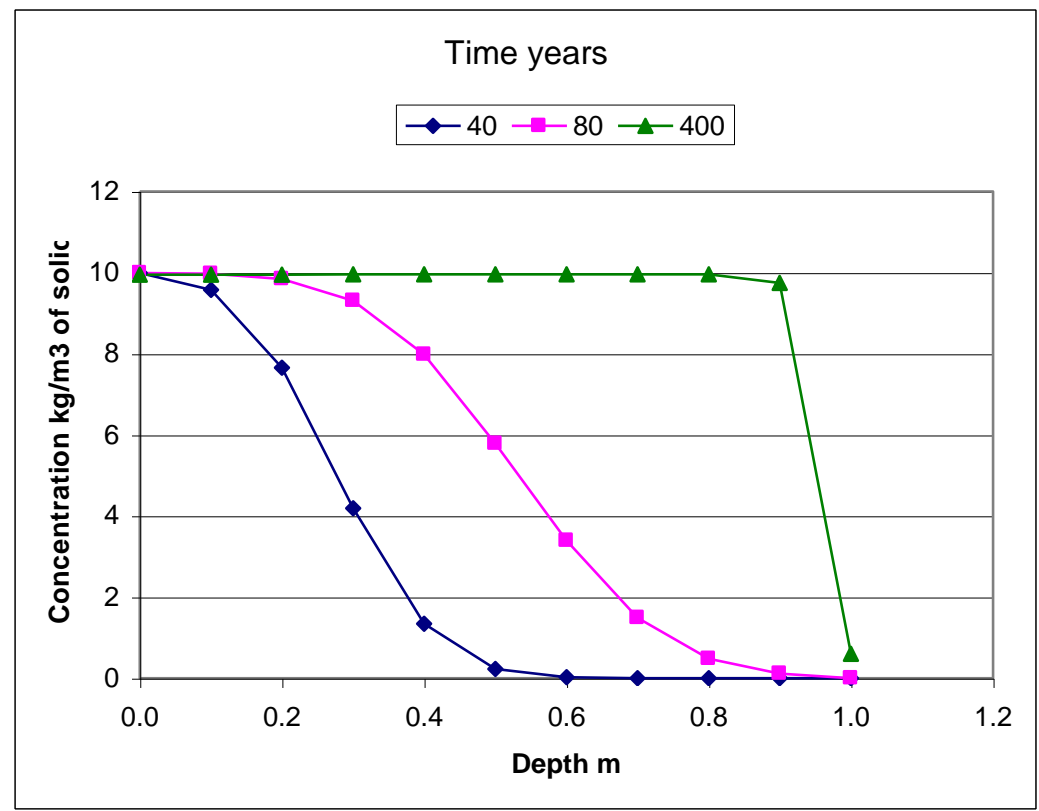

Figure 8. Concentrations of contaminants for base case 


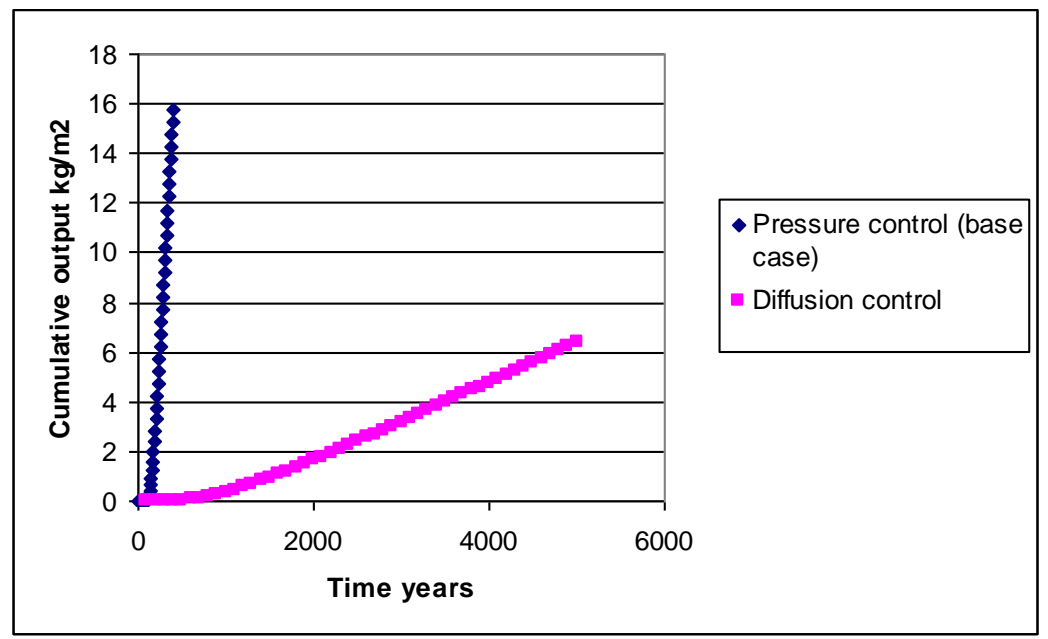

Figure 9. Output of contaminants from the base of the barrier

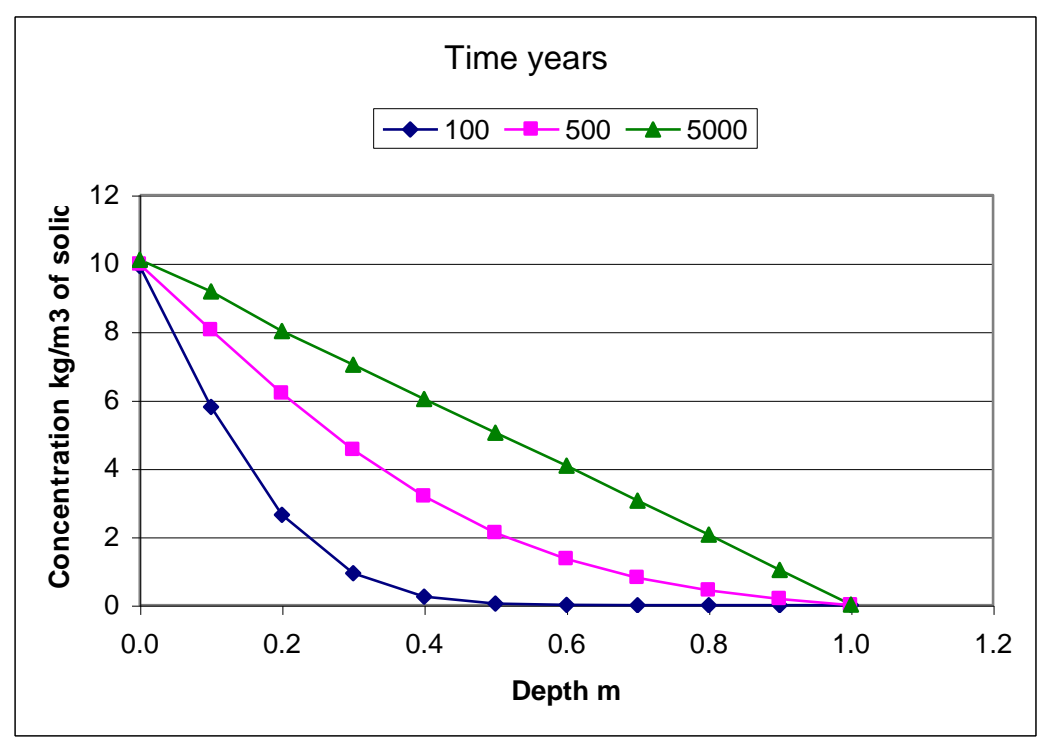

Figure 10 Concentrations of contaminants for diffusion control 


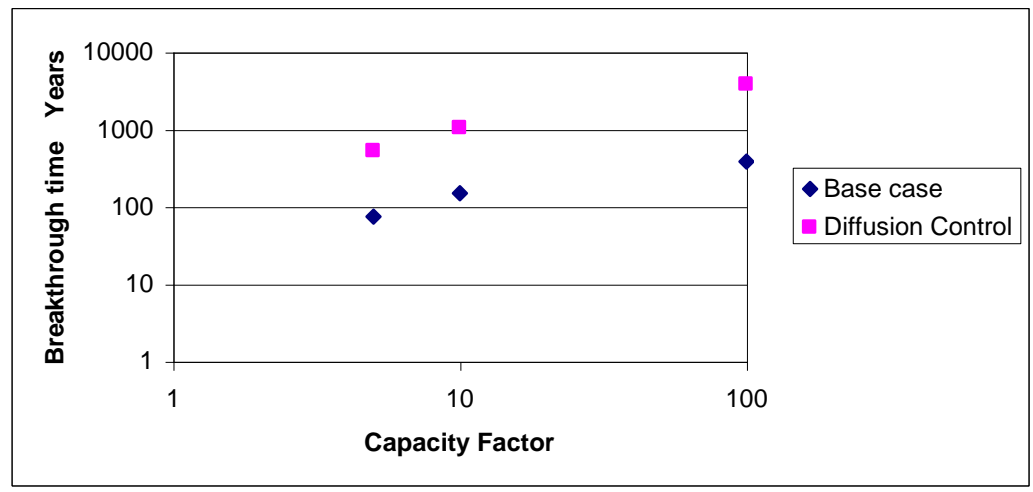

Figure 11 . The effect of capacity factor. 